\title{
ASPECTOS DE LA DISTRIBUCION ALGAL EN EL LITORAL GRANADINO
}

\author{
J. VARO y J. RAMIREZ*
}

\section{RESUMEN:}

Como un paso previo al establecimiento de la fitosociología de la flora bentónica en el litoral grandino, se describen las características fisionómicas de las poblaciones en zonas de actividad hidrodinámica, iluminación, sustrato, etc. . . diferentes.

Mediante criterios cualitativos de presencia y abundancia, se determinan las preferencias de las diferentes especies por un determinado nivel, en lo referente a la zonación vertical. Los cuadros elaborados ponen de manifiesto la delimitación de poblaciones litorales e infralitorales bién definidas, si bién, en el horizonte medio de la zona litoral, la vegetación debe ser considerada como de tránsito entre la de los horizontes superior e inferior, resultado de la escasa amplitud de las mareas mediterráneas.

\section{SUMMARY:}

In the present paper are described the fisionomics characteristics of the benthonic seaweeds populations in places of different hidrodynamic activity, illumination, substratum, etc... We have elaborated tables of the specific taxons which are set, on differents levels, in a vertical zonation.

Ante la iniciativa del Departamento de Botánica de la Universidad de Granada, de estudiar de la forma más completa posible la diversidad florística de la provincia dirigimos nuestra atención al capítulo de la vegetación bentónica. El presente trabajo, llevado a cabo durante los años 1975 a 1977, ha tenido como objetivo introducirnos en este aspecto de la Botánica y fruto del mismo ha sido la confección del catálogo florístico y la obtención de datos sobre la distribución de las diferentes especies inducida por agentes ecológicos tales como hidrodinamismo, iluminación, sustrato, etc., y cuyo desglosamiento constituye el contenido del trabajo que presentamos. 
A su realización nos ha movido el gran interés que despierta actualmente el estudio de la biocenosis del bentos marino, induciendo a un número elevado de especialistas a encaminar sus estudios al tema de la fracción algal, como componentes fundamentales de las comunidades bentónicas, así como establecer los patrones fitosociológicos que las delimitan.

Es precisamente la ficosociología el aspecto más polémico ya que no se han elaborado aún, de forma unitaria, los criterios que han de determinar el acotamiento de las poblaciones, utilizándose procedimientos muy variados, bien basados en esquemas desarrollados para ámbitos terrestres, o bien con métodos individuales fruto de la propia iniciativa de los estudiosos del tema.

Se hace preciso pues enfrentarse con el estudio de las poblaciones algales, tratando de solventar los problemas que actualmente se plantean. Somos conscientes, sin embargo, de las dificultades que entraña tal propósito, por lo que hemos considerado como un primer paso encaminar nuestro estudio al conocimiento de la distribución de las especies en función de los factores ecológicos que inciden en ellas, incluyendo la descripción de las poblaciones características a fin de establecer una base que sustente posteriores estudios de matiz fitosociológico.

Se han obtenido inventarios en cada uno de los puntos observados atendiendo a iluminación, condiciones hidrodinámicas, naturaleza y morfología del sustrato sobre el que se asientan, nivel de la costa que ocupan, etc., introduciendo en bolsas de plástico las especies encontradas en cada punto de inventario.

Tras la realización del catálogo florístico, que se desarrolló a lo largo de 12 estaciones y a tenor de las frecuentes visitas que fueron practicadas, nocreímos necesario extender la toma de inventarios a todas ellas, reduciéndolas a 5: Cerro Gordo, Los Berengueles, Peñones del Santo, Calahonda y Punta del Melonar, que reunen las diversas características ecológicas observadas en nuestra zona y la totalidad de las especies que habían sido catalogadas.

Los métodos que se exponen en la bibliografía para la toma de inventarios son muy diversos, radicando la mayor discrepancia en la concepción de los índices que han de determinar las comunidades algales. Como el propósito de este trabajo no es delimitar fitosociológicamente las poblaciones, hemos renunciado a considerar tales índices, si bién se establece, en nuestros inventarios, un criterio cualitativo de presencia necesario para obtener las poblaciones características en cada zona. El índice de presencia señala, en un baremo de 0 a 5 , el número de estaciones en que ha sido encontrada una determinada especie, en un nivel considerado.

En cada una de las cinco estaciones se han tomado los inventarios atendiendo al siguiente esquema:

Poblaciones Supralitorales

Poblaciones Litorales en el Horizonte Superior

Poblaciones Litorales en el Horizonte Medio

Poblaciones Litorales en el Horizonte Inferior

Poblaciones Infralitorales, fondo de 0 a $4 \mathrm{mts}$.

Poblaciones Infralitorales, fondo de $4 \mathrm{mts}$. en adelante 
En recorridos de superficie y de fondo (valiéndonos de equipos de inmersión) tomábamos nota de la evolución de las poblaciones dependiente de las condiciones ecológicas cambiantes, a lo largo de cada zona.

Los factores ecológicos han sido considerados de una forma cualitativa ante la imposibilidad de disponer de aparatos de medida necesarios, que porotra parte excederían el propósito del presente trabajo.

La nota característica del litoral estudiado, como corresponde a todo aquel bañado por masas de agua cerradas, es la escasa amplitud de las mareas, fenómeno que unido al hecho de la predominancia del sustrato móvil frente al rocoso, hace que los diferentes niveles a considerar en la distribución vertical queden muy desdibujados, solapándose frecuentemente, sobre todo en la Zona Litoral, en donde la mayoría de las veces se hace imposible distinguir los tres niveles distintos, ajustándose la distribución al criterio propuesto por Seoane Camba.

\section{Zona Supralitoral.}

Se muestra desprovista de vegetación algal en su mayor parte, si bién en aquellos lugares donde el hidrodinamismo asegura una zona de humectación mínima, producida por la salpicadura contínua del oleaje, aparece Bangia fuscopurpurea, formando típicos mechones que coronan el roquedo, como es el caso de los Peñones del Santo donde en algunos sitios llega a situarse hasta un metro por encima del nivel del mar, coincidiendo con un batimiento extremo y siempre en la superficie de las rocas que reciben directamente las salpicaduras.

En localidades semibatidas, confundiéndose con el Horizonte Superior de la Zona Litoral, también aparece esta especie, en bandas muy estrechas. Sin embargo, B. fuscopurpurea no ha sido considerada como representativa del Supralitoral por dos razones:

En primer lugar el hecho de aparecer en sitios donde el hidrodinamismo es acentuado puede hacer pensar en una ampliación del Horizonte Superior de la Zona Litoral, corroborado al mostrarse confundida con su vegetación en lugares de batimiento más atenuado.

En segundo lugar por ser más frecuente y aparecer de forma más abundante en zonas netamente litorales.

\section{Zona Litoral.}

En las regiones mediterráneas es sin duda la que presenta una mayorcomplejidad en la zonación de sus elementos por la fluctuación poco amplia de las mareas.

En nuestra costa, el Horizonte Superior aparece poblado por una vegetación cespitosa, fundamentalmente constituída por Gelidium crinale, en la que destacan como especies características las consignadas en el Cuadro I. 
En lugares batidos y semibatidos, la capa de G. crinale se hace discontinua siempre que no se sitúe sobre un estrato calcáreo constituído por corallináceas que le proporcionan una considerable consistencia, en cuyo caso forma tapiz continuo donde es posible encontrar agregados de Laurencia obtusa, Laurencia pinnatifida y destacando sobre él, en los lugares más expuestos, frondes de Rissoella verruculosa, endemismo silicícola mediterráneo, según apunta Molinier, y Nemalion helminthoides. En zonas muy batidas de los Peñones del Santo hemos observado Rissoella verruculosa a una altura superior, a un metro por encima del nivel del mar.

En lugares de batimiento menos acentuado se sitúa una banda de Ulva rigida que queda por encima de la de $G$. crinale en superficies verticales obién se superpone, en superficies de menor inclinación, formando una población densa en lugares protegidos que se hace más diseminada cuando el hidrodinamismo aumenta, en este caso $U$. rigida necesita una mayor protección que busca entre la vegetación circundante o bién entre las grietas del roquedo.

Frecuentemente acompañan a estas especies Enteromorpha compressa y Cladopbora laetevirens, aún cuando la primera se encuentra más asiduamente en cubetas y depresiones algo batidas y la segunda tiene posibilidad de bajar a niveles inferiores mezclándose con Corallina mediterranea.

De forma muy escasa ha sido encontrado Caulacanthus ustulatus contribuyendo al porte cespitoso característico de esta población, de igual forma que ocurre con Gigartina acicularis.

Porphyra umbilicalis aparece en lugares semibatidos, desapareciendo durante los meses estivales.

En las repisas igualmente semibatidas que se producen por efecto de la erosión, la vegetación se hace muy densa, siendo posible advertir una gran cantidad de Cladophora laetevirens y Cladophora lebmanniana, que se entremezclan con el césped ya descrito.

Un capítulo importante en este horizonte es la vegetáción de cubetas, lugares donde queda estancada el agua bien en la bajamar o bien por efecto de las salpicaduras, que es el caso más frecuente en el litoral granadino. Son pocas las que se encuentran totalmente desligadas del mar puesto que la proximidad de la superficie las hace vulnerables. Aún así en Calahonda y especialmente en los Peñones del Santo es posible encontrar cubetas propiamente dichas que reunen las condiciones de aislamiento requeridas.

La vegetación que integra tales hábitats muestra una gran abundancia de Chaetomorpha aerea y Cladophora albida, en masas enmarañadas, así como Cladophora sericea, Enteromorpha compressa y Enteromorpha intestinalis en menor cantidad.

En las cubetas donde el agua resulta algo movida por efecto del oleaje y que se han considerado como depresiones a fin de diferenciarlas de las propiamente dichas, la vegetación es tanto más variada cuanto mayor es la agitación, mostrándose abundantes Enteromorpha compressa y Ulva rígida e introduciéndose en ellas Cladopbora rupestris, Cladophora laetevirens y Polysiphonia opaca, que se sitúan en los bordes de la depresión, muy próximas al horizonte medio y que tienden a integrarse ya en su vegetación característica. 
En algunas localidades entre los horizontes superior e inferior de esta zona, se extiende una vegetación mixta en la que parece delimitarse especies que podrían ser características de un horizonte medio, pero siempre muy distinto al oceánico. La fisionomía que presenta está relacionada muy directamente con las condiciones hidrodinámicas del lugar, haciendo predominar especies de uno y otro horizontes.

En localidades semibatidas o calmas continúa la vegetación cespitosa del litoral superior con características muy similares a las descritas, apareciendo Gelidium crinale algo más escaso, junto con Laurencia pinnatifida, Laurencia obtusa, Gigartina acicularis, Cladophora pellucida, Chaetomorpha linum y Corallina mediterranea, con mas frecuencia está última que en el horizonte superior. Más dispersas aparecen Ulva rigida, Caulacanthus ustulatus, Colpomenia sinuosa y típicamente embutida en la vegetación Valonia utricularis. En ambientes esciófilos representados a este nivel por grietas y oquedades, se han observado Cladophora prolifera, Gymnogongrus patens, Dictyopteris polipodioides y Peyssonelia coriacea.

A medida que la intensidad hidrodinámica aumenta se va operando un cambio de fisionomía comenzando a dominar algas calcáreas como Corallina mediterranea, primordialmente, Lithophyllum incrustans y algo más escasa Mesophyllum lichenoides, mientras que la vegetación cespitosa se enmascara entre la calcárea contituyéndose agregados muy compactos de Gelidium crinale, Corallina mediterranea, Cladophora laetevirens, Polysiphonia pennata y Gigartina acicularis y epífitos como Polysiphonia fruticulosa, que alternan con céspedes de ceramiales integrados por $\mathrm{Ce}_{e}$ ramium flabeligerum, C. ciliatum y C. rubrum, muy abundantes, junto con Polysiphonia nigrescens y Callithamnion granulatum.

Las especies reseñadas en el Cuadro II son las que de una forma difusa pueden caracterizar un horizonte medio de naturaleza netamente mediterránea, pero como se desprende del estudio de la población a este nivel, la actividad hidrodinámica influye de forma que la vegetación adopta características bien del horizonte superior, bien del inferior, de tal manera que a nuestro juicio es correcto suponer que representa en realidad una zona de tránsito entre ambos niveles. Refiriéndose de nuevo al Cuadro II se ha de puntualizar que algunas especies no aseguran su carácter de horizonte medio, puesto que han sido encontradas en una sóla ocasión, lo que impide discutir su comportamiento.

El poblamiento del Horizonte inferior se perfila en nuestro litoral muy bien definido y continuo, en el que las algas calcáreas constituyen la base de la vegetación, destacando sobre el resto de las especies en mayor o menor grado, según el hidrodinamismo del lugar.

En zonas calmas o semibatidas, sobre capas de algas calcáreas laminares como Lithophyllum incrustans o Mesophyllum lichenoides, se dispone una vegetación densa constituída por céspedes de Caulacanthus ustulatus, Gigartina acicularis, Cladophora pellucida, Gelidium latifolium, Corallina mediterranea (escasa) y Cladophora laetevirens que alternan con densos grupos de Ceramium diaphanum y Ceramium rubrum. De forma dispersa se observa Valonia utricularis y Colpomenia sinuosa, más abundantes, sin embargo, que en los niveles anteriores, así como Bryopsis plumosa y Ulva rigida. 
En aquellos lugares de iguales condiciones hidrodinámicas pero protegidos de una iluminación intensa se han encontrado Sargassum vulgare, Dictyopteris polipodioides, Gymnogongrus patens, Sphacelaria plumula y Sphacelaria pennata, frecuente esta última como epifita sobre Peyssonelia coriacea, Stypocaulon scoparium y Cystoseira ericoides.

En superficies verticales semibatidas se disponen capas de Codium adhaerens a veces muy extensas, que son desplazadas en sustratos horizontales por Cystoseira ericoides. En ocasiones $C$. adhaerens sube de nivel entrando a formar parte de la vegetación mixta superior. Al aumentar las condiciones hidrodinámicas se produce un retraimiento de la vegetación cespitosa cuyas especies se disponen, bien por encima de las corallináceas que se hacen dominantes, bien bajan a la Zona Infralitoral, (las que como Gelidium latifolium son también propias de tal zona), o bien se protegen entre las corallináceas, como sucede en el horizonte medio.

El desarrollo en los litorales expuestos de este horizonte, de Corallina mediterranea, Lithophyllum incrustans, Mesophyllum lichenoides, Amphiroabeauvoisii y Lithophyllum tortuosum es tal que constituyen verdaderas cornisas de sustrato sólido, denominadas en la bibliografía como "Trottoir», típicos del Mediterráneo Occidental, que permiten la supervivencia de especies que se entrelazan densamente con los talos de Corallina o Amphiroa, o bien son cubiertos por las expansiones laminares de Mesophyllum, adentrándose hasta lugares donde el hidrodinamismo es tan intenso que sólo permite la existencia de calcáreas, así como talos rastreros y enmarañados de Gigartina acicularis.

Algunas especies como Cystoseira ericoides recurren a una disminución de su porte para soportar el batimiento, habiendo sido encontrados talos pequeños y dispersos en los lugares más expuestos de los Peñones del Santo, que es sin duda la localidad que muestra mayor actividad hidrodinámica en nuestro litoral.

La vegetación esciófila está representada, en esta zona, por Plocamiun coccineum y Peyssonelia coriacea, fundamentalmente en los lugares semibatidos.

A pesar de la diversidad que muestra el horizonte inferior, su vegetación muestra una fisionomía muy característica perfectamente diferenciable de la del horizonte superior. Las especies que han mostrado una constancia exclusiva o mayor que las demás en este horizonte, se encuentran reseñadas en el Cuadro III.

\section{Zona Infralitoral.}

Se han distinguido en esta zona dos niveles, variables en cuanto al área que comprenden, uno, la región que afecta el rompeolas y otro el que queda por debajo de ella, hasta la profundidad máxima muestreada.

En el primero es posible advertir una sucesión rápida de la vegetación litoral hacia el establecimiento de otra típicamente infralitoral, expuesta en el Cuadro IV.

En zonas poco batidas el tránsito es rápido, apareciendo en primer lugar una banda densa de Gelidium latifolium, Pterocladia capillacea, Codium adhaerens, Codium tomentosum y Colpomenia sinuosa, que en superficies horizontales cubre grandes extensiones, y al ser especies de desarrollo considerable permiten la exis- 
tencia de algas esciófilas como Peyssonelia coriaceay Plocamium coccineum, mostrándose esta última con preferencia por regiones más movidas.

Al aumentar el hidrodinamismo Pterocladia capillacea es desplazada quedando Gelidium latifolium como especie mayoritaria, además de Corallina mediterranea y Mesophyllum lichenoides, vegetación que se ve incrementada por Asparagopsis armata y Falkembergia rufolanosa, cuando el movimiento del agua se traduce por un vaivén más que por un choque directo. Frecuentemente Pterocladia capillacea aparece con las porciones basales calcificadas por la epifita Melobesia sp., soportando entonces situaciones de más batimiento.

Cystoseira ericoides y Cystoseira fimbriata alcanzan un porte considerable en zonas preferentemente horizontales y semibatidas. Dictyota dichotoma, Padina pavonia, Stypocaulon scoparium, Halopteris filicina y Zonaria tournefortii marcan el límite entre los dos niveles establecidos en la Zona Infralitoral.

En zonas muy expuestas la vegetación calcárea que se sitúa en el horizonte inferior baja con iguales características a la Zona Infralitoral, destacando Corallina mediterranea en formaciones muy compactas en aquellos lugares de choque intenso del oleaje o en oquedades donde al actuar éste a modode pistón produce un gran efecto de compresión, mientras que son las calcáreas foliosas como Lithophyllum o Mesophyllum las que se muestran más aparentes cuando el batimiento se produce por reflexión del oleaje, de forma unidireccional. En una u otra situación algas como Gelidium latifolium y Cystoseira ericoides aparecen muy protegidas, dispersas y de talo reducido, apreciándose una importante vegetación epifita constituída por Apoglossum ruscifolium, Anthithamnion plumula, Anthithamnion cruciatum, Rhodymenia palmetta, Herposiphonia secunda y Dasya arbuscula, muy frecuentes sobre Corallina mediterranea.

Por debajo de esta zona batida se sitúa la vegetación de Pterocladia capillacea y Gelidium latifolium, mencionada para las localidades menos expuestas, con la mayoría de sus integrantes, si bien es de destacar el hecho de que al estar tamizada la iluminación del medio, las especies esciófilas, relegadas en los niveles superiores a grietas y oquedades, salen de éstos cubriendo amplias zonas de sombra haciendo posible encontrar grandes extensiones de Plocamium coccineum y Plocamium rafelisianum en lugares movidos y en sitios más tranquilos junto con Peyssonelia coriacea, Lithophyllum incrustans, Sphacelaria plumula y Sphacelaria pennata.

En las regiones no influenciadas por el rompeolas, donde las corrientes son las únicas responsables del movimiento, domina una vegetación homogénea. Sólamente en dos estaciones ha sido posible seguir la distribución de las algas hasta un fondo de $20 \mathrm{mts}$., Calahonda y Los Berengueles, pues lo normal es que el roquedo se circunscriba a las proximidades de la costa, hasta una profundidad de 8 a $10 \mathrm{mts}$. a partir de la cual se extiende el sustrato móvil, donde de forma esporádica se disponen Cladostephus verticillatus y Dictyota dichotoma.

La vegetación que se sitúa sobre superficies horizontales, mayoritariaen nuestro litoral, está integrada por Stypocaulon scoparium, Cladostephus verticillatus y $\mathrm{Ha}$ lopteris filicina, que alcanzan una cobertura considerable, junto a Cystoseira ericoides, Padina pavonia, Dictyota dichotoma y Codium tomentosum, como especies que muestran un mayor desarrollo del talo. Entre ellas se disponen Jania rubens, Jania longi- 
furca, talos dispersos de Gelidium latifolium, Asparagopsis armata y mostrándose escasas Corallina mediterranea y Sphaerococcus coronopifolius. Componiendo un estrato laminar que recubre las piedras, a veces en capas contínuas, sobre el que se dispone la vegetación antes descrita, han sido encontradas las especies Lithophyllum tortuossum, Lithophyllum incrustans y Lithophyllum dentatum, así como Peyssonelia atropurpurea, en costras más reducidas y sólo a partir de $10 \mathrm{mts}$. de profundidad.

Las superficies verticales son mas escasas en estos fondos, generalmente localizadas en las proximidades de la costa. En ellas se sitúan Codium difforme, Codium adhaerens y Codium bursa, así como otras especies que se comportan de forma indiferente respecto al sustrato como Gelidium latifolium, Jania rubens y Stypocaulon scoparium, entre otras.

Las poblaciones epifitas se disponen preferentemente sobre los talos más frondosos, habiéndose determinado Catenella repens, Sphacelaria pennata, Ceramium echionotum, Ceramium diaphanum y Apoglossum ruscifolium.

A partir de los $10 \mathrm{mts}$. , de profundidad se observa una disminución de la cobertura haciéndose más aparentes las costras calcáreas, antes mencionadas, apareciendo de forma esporádica Halopteris filicina, Stypocaulon scoparium, Cladostephus verticillatus y Peyssonelia atropurpurea.

Por último en los cuadros V y VI, se reseñan las especies que muestran una tendencia generalizada hacia las Zonas Litoral e Infralitoral, respectivamente, y en el VII, aquellas que se comportan como cosmopolitas. 
CUADRO I

CONSTANCIAS DE APARICION DE LAS ESPECIES MAS

REPRESENTATIVAS DEL horizonte superior DE LA

ZONA LITORAL

\begin{tabular}{lcccc}
\hline & H S & H M & H I & I \\
\cline { 2 - 5 } Enteromorpha clathrata & 2 & 1 & 0 & 0 \\
Bangia fuscopurpurea & 4 & 0 & 0 & 0 \\
Nemalion helminthoides & 2 & 1 & 0 & 0 \\
Rissoella verruculosa & 1 & 0 & 0 & 0 \\
\hline
\end{tabular}

CUADRO II

CONSTANCIAS DE APARICION DE LAS ESPECIES MAS

REPRESENTATIVAS DEL horizonte medio DE LA

ZONA LITORAL

Ulva lactuca

Polysiphonia pennata

Ceramium flabeligerum

Ceramium rubrum

Chondria coerulescens

Spyridia filamentosa

Polysiphonia opaca

Polysiphonia nigrescens

Acrosorium uncinatum

Spirulina subsalsa

Oscillatoria subuliformis

Caulacanthus ustulatus

\begin{tabular}{cccc} 
H S & H M & H I & I \\
\hline 0 & 1 & 0 & 0 \\
0 & 1 & 0 & 0 \\
0 & 1 & 0 & 0 \\
0 & 4 & 3 & 0 \\
0 & 1 & 0 & 0 \\
0 & 1 & 0 & 0 \\
0 & 3 & 0 & 0 \\
0 & 3 & 1 & 1 \\
0 & 1 & 0 & 0 \\
0 & 2 & 0 & 0 \\
0 & 1 & 0 & 0 \\
1 & 2 & 0 & 0
\end{tabular}

CUADRO III

CONSTANCIAS DE APARICION DE LAS ESPECIES MAS

REPRESENTATIVAS DEL horizonte inferior DE LA

ZONA LITORAL

Bryopsis plumosa

Sargassum vulgare

Polysiphonia thuyoides

Gymnogongrus patens

\begin{tabular}{cccc} 
H S & H M & H I & I \\
\hline 0 & 0 & 1 & 0 \\
0 & 0 & 1 & 0 \\
0 & 1 & 2 & 0 \\
0 & 1 & 2 & 0
\end{tabular}




\section{CUADRO IV \\ CONSTANCIAS DE APARICION DE LAS ESPECIES MAS REPRESENTATIVAS DE LA ZONA INFRALITORAL}

\begin{tabular}{lcccc}
\hline & H S & H M & H I & I \\
\cline { 2 - 5 } Bryopsis bipnoides & 0 & 0 & 1 & 3 \\
Cladostephus verticillatus & 0 & 0 & 0 & 5 \\
Codium bursa & 0 & 0 & 0 & 5 \\
Codium tomentossum & & & & \\
Codium tomentosum & 0 & 0 & 0 & 5 \\
Codium difforme & 0 & 0 & 0 & 2 \\
Colpomenia peregrina & 0 & 0 & 0 & 1 \\
Dictyota dichotoma & 0 & 0 & 0 & 5 \\
Padina pavonia & 0 & 0 & 0 & 4 \\
Zonaria tournefortii & 0 & 0 & 0 & 1 \\
Lithophyllum tortuosum & 0 & 0 & 0 & 1 \\
Lithophyllum dentatum & 0 & 0 & 0 & 1 \\
Ceramium echionotum & 0 & 0 & 0 & 3 \\
Herposiphonia secunda & 0 & 1 & 0 & 2 \\
Peyssonelia atropurpurea & 0 & 0 & 0 & 1 \\
Plocamium rafelisianum & 0 & 0 & 0 & 1 \\
Gastroclonium ovale & 0 & 0 & 0 & 1 \\
Anthithamnion cruciatum & 0 & 0 & 0 & 1 \\
Anthithamnion plumula & 0 & 0 & 1 & 4 \\
Dasya arbuscula & 0 & 0 & 0 & 2 \\
Oscillatoria bonemaissonii & 0 & 0 & 0 & 1 \\
Oscillatoria corallinae & 0 & 0 & 0 & 2 \\
\hline
\end{tabular}

Los cuadros 5, 6 y 7 representa aquellas especies que muestran un comportamiento generalizado en cuanto al lugar que ocupan.

CUADRO V

ESPECIES QUE MUESTRAN TENDENCIA A LA ZONA LITORAL CON INDICES CONSTANTES

\begin{tabular}{lcccc}
\hline & H S & H M & H I & I \\
\cline { 2 - 5 } Cladophora albida & 2 & 2 & 1 & 0 \\
Cladophora sericea & 2 & 1 & 1 & 0 \\
Chaetomorpha aerea & 1 & 1 & 0 & 0 \\
Chaetomorpha aerea & 1 & 1 & 0 & 0 \\
Enteromorpha compressa & 3 & 3 & 1 & 0 \\
Enteromorpha ramulosa & 1 & 1 & 0 & 0 \\
Rhizoclonium riparium & 1 & 1 & 0 & 0 \\
Ulva rigida & 5 & 5 & 3 & 0 \\
Gelidium crinale & 5 & 5 & 2 & 0 \\
Polysiphonia fruticulosa & 2 & 3 & 3 & 0 \\
Polysiphonia nigrescens & 0 & 3 & 1 & 1 \\
Jania longifurca & 0 & 1 & 1 & 0 \\
Callithamnion granulatum & 0 & 3 & 3 & 1 \\
Laurencia pinnatifida & 4 & 5 & 3 & 0 \\
Laurencia obtusa & 3 & 4 & 3 & 0 \\
Rivullaria bullata & 1 & 1 & 0 & 0 \\
\hline
\end{tabular}


CUADRO VI

ESPECIES QUE MUESTRAN TENDENCIA A LA ZONA

INFRALITORAL CON INDICES CONSTANTES

\begin{tabular}{lcccc}
\hline & H S & H M & H I & Z I \\
\cline { 2 - 5 } Bryopsis balbisiana & 0 & 1 & 1 & 2 \\
Cladophora pellucida & 0 & 2 & 1 & 4 \\
Cladophora prolifera & 0 & 2 & 2 & 4 \\
Codium adherens & 0 & 2 & 3 & 2 \\
Colpomenia sinuosa & 0 & 2 & 3 & 4 \\
Cystoseira fimbriata & 0 & 0 & 1 & 1 \\
Cystoseira ericoides & 0 & 2 & 3 & 5 \\
Dictyopteris polipodioides & 0 & 1 & 1 & 1 \\
Valonia utricularis & 0 & 4 & 5 & 2 \\
Sphacelaria pennata & 0 & 0 & 2 & 5 \\
Sphacelaria plumula & 0 & 0 & 1 & 3 \\
Polisiphonia macrocarpa & 2 & 3 & 2 & 4 \\
Peyssonelia coriacea & 0 & 2 & 5 & 5 \\
Rhodymenia palmetta & 0 & 3 & 3 & 5 \\
Mesophyllum lichenoides & 0 & 3 & 3 & 4 \\
Pterocladia capillacea & 0 & 3 & 2 & 5 \\
Jania rubens & 0 & 2 & 3 & 5 \\
Amphiroa beauvoisii & 0 & 1 & 2 & 2 \\
Asparagopsis armata & 0 & 2 & 2 & 5 \\
Falkembergia rufolanosa & 0 & 2 & 2 & 5 \\
Ceramium ciliatum & 0 & 3 & 3 & 4 \\
Ceramium diaphanum & 0 & 1 & 3 & 4 \\
Gelidium latifolium & 0 & 4 & 5 & 5 \\
Plocamium coccineum & 0 & 4 & 5 & 5 \\
Catenella repens & 0 & 1 & 1 & 4 \\
Apoglossum ruscifolium & 0 & 2 & 1 & 5 \\
Spondilothamnion multifidum & 0 & 1 & 2 & 4 \\
Lithophyllum incrustans & 0 & 2 & 3 & 5 \\
\hline & & & &
\end{tabular}

\section{CUADRO VII \\ ESPECIES COSMOPOLITAS CON INDICES ALTOS EN \\ TODAS LAS ZONAS}

\begin{tabular}{lcccc}
\hline & H S & H M & H I & Z I \\
\cline { 2 - 5 } Cladophora laeterirens & 5 & 4 & 4 & 3 \\
Cladophora lehmanniana & 3 & 2 & 2 & 1 \\
Cladophora rupestris & 2 & 1 & 1 & 0 \\
Chaetomorpha linum & 4 & 3 & 3 & 2 \\
Corallina mediterranea & 5 & 5 & 5 & 4 \\
Gigartina acicularis & 2 & 5 & 5 & 5 \\
Enteromorpha intestinalis & 2 & 2 & 0 & 1 \\
\hline
\end{tabular}




\section{BIBLIOGRAFIA}

ARDRE, F. 1971. Contribution á l'etude des algues marines du Portugal. Bull. Cent. Etude. Rech. Sci. 8 (3): 359-574.

BOUDOURESQUE, C. 1970. Recherches de bionomie analytique, structurale et expérimentale sur les peuplements benthiques sciéphiles de Méditerranée occidentale (fraction algale). Tesis Doctoral.

CODO MIER, L. 1974. Recherches sur les kallymenia (Cryptonemiales, kallymeniacées). Vie et Milieu, 24 (3): 369-388.

CODOMIER, L. 1974. Recherches sur la structure et le développement des Halymenia C. Ag. (Rhodophycées, Cryptonémiales) des côtes de France et de la Méditerranée. Vie et Milieu, 24 (1): 1-42.

GAYRAL, P. 1958. Algues de la côte atalantique marocaine. Ed. du Casablanca. Rabat.

GAYRAL, P. 1966. Les algues des côtes francaises. Ed. Doin. Paris.

GONZALEZ GUERRERO, P. 1950. Hallazgos ficológicos en nuestra costa. A nal. Inst. Bot. Cavanilles, 10: 191-230. Madrid.

GONZALEZ GUERRERO, P. 1952. El epifitismo ficológico en el Mar Menor (Murcia). A nal. Inst. Bot. Cavanilles, 11 (1): 143-151. Madrid.

HAMEL, G. \& LEMOINE, P. 1952. Corallinacées de France et D’Afrique du Nord. Arch. du Mus. Nat., 7. ${ }^{a}$ 1: 17-131. Paris.

KNOEPFFLER-PEGUY, M. 1974. Le genre A cinetospora Bornet 1891 (PhaeophyceaeEctocarpales). Vie et Milieu, 24 (1): 43-72.

MARGALEF, R. 1974. Ecología. Ed. Omega. Barcelona.

MENDEZ DOMINGO, C. 1955/56. Datos ecológicos de las algas marinas de Torrevieja (Alicante). A nal. Inst. Bot. Cavanilles, 14: 145-151. Madrid.

MOLINIER, R. \& VIGNES, P. 1976. Introducción a la Ecobiocenología. Ed. Vicens Vives. Barcelona.

NEWTON, L. 1931. A handbook of the British Seaweeds. Londres.

PEREZ-CIRERA, J. L, 1975. Catálogo florístico de las algas bentónicas de la Ría de Cedeira, NO. de España. Anal. Inst. Bot. Cavanilles, 32 (2): 53-74. Madrid.

RAMIREZ RODRIGO, J. 1977. Poblamiento algal de la costa granadina: Algas verdes y pardas. Manuscrito.

RENTERIA SARMIENTO, J. 1977. Algas rojas y azules del litoral granadino. Manuscrito.

SEOANE-CAMBA, J. 1965. Estudios sobre las algas bentónicas en la costa sur de la Península Ibérica (litoral de Cádiz). Inv. Pesq., 29. Barcelona.

- 1967. La explotación de las algas mannas. Ink. Inv. Pesq. Barcelona.

- 1967. Las especies españolas de Gigartina y Chondrus: El Carragaen. Inst. Inv. Pesq., 6: 291-302. Barcelona.

- 1969. Crecimiento, producción y desprendimiento de biomasa en Gelidium sesquipadale (Cle.) Thuret. Proc. Intl. Seaweed Symp., 6: 365-374.

- 1975. Algas bentónicas españolas en los herbarios Thuret-Bonet y Sauvageau del Museum National d'Histoire Naturelle de Paris. Anal. Inst. Bot. Cavanilles, 32 (2): 3351. Madrid.

- 1975. Perspectivas en el aprovechamiento de los recursos marinos. Rev. Fac. Farm.

- 1975. Características de la vegetación marina en la costa de Guipuzcoa. Anal. Inst. Bot. Cavanilles, 32 (1): 173-183. Madrid.

- 1977. Sur une nouvelle espéce de Gelidiella trouvée aux Iles Canaries: Gelidiella tinerfensis nov. sp., Soc. Pbycol. de France, Bull., 22: 127-133.

- \& POLO, L. 1974. Estudio botánico de la plataforma submarina catalana. Anal. Inst. Bot. Cavanilles, 31 (1): 179-183. Madrid.

VAN DEN HOEK, C. 1963. Revision of the European speces of Cladophora. Ed. Otto Koeltz Science Publishers. Leiden. 